\title{
Data Preprocessing Circuit Designs and Analyses for Subsonic Cruise Mis- sile Infrared Image Seeker
}

\author{
Tain-Sou Tsay*
}

Department of Aeronautical Engineering, National Formosa University, Yunlin, Taiwan

\begin{abstract}
In this literature, a data pre-processing circuit designs and analyses for an infrared image seeker of a subsonic cruise missile are proposed. The flight speed of the missile is $250 \mathrm{~m} / \mathrm{s}$. Therefore, automatic contrast control and automatic brightness control are usually needed to keep wanted contrast and brightness for best image properties in target recognition and tracking; especially for the missile at the terminal approaching phase to prevent signal saturation. Signal saturation implies target lost. The major parameters of imaging systems for contrast and brightness controls include average, maximum and minimum of gray level of the whole picture in the sampling interval. Therefore, the overall system is a complicated nonlinear sampled-data control system. It is difficult to describe, analyze and design the system. In this literature, the overall system is first decomposed into two linearized sampled-data control systems to get needed loop compensations, and verified by a special range-dependent testing signal to the overall system secondly. The controlled system is further verified by a thermal plate and real flight testing. Testing results give the proposed method can provide effective way to analyze and design the considered system.
\end{abstract}

\section{INTRODUCTIONS}

Multiple sensors for ECCM are generally expected for missile homing systems. The first dual seeker application of the world for subsonic antiship missile is the HF-2 developed in Taiwan. It includes a Radar seeker and an IR image seeker with data fusing algorithm. This application prompts the capability for ECCM from chaff jamming to RF seeker and thermal jamming to IR Image seeker. The problem for IR image seeker for the missile application is the thermal energy to the charge-coupled device (CCD) [1-3] is the fourth power of the reciprocal the distance between image seeker and thermal source; i.e.; missile and target. It needs faster automatic gain control (AGC) for contrast and automatic pedestal control (APC) for brightness [4-7] to keep best imaging properties for target recognition and tracking; especially for the missile at terminal approaching phase. Slower response for coping with thermal energy increasing will make the signal output be saturated; i.e., all white of full frame and will lost tracking centre point for missile guidance.

It this literature, models for contrast, brightness and dynamic signals are proposed to analyses and designs of the AGC and APC circuit loops. The AGC and APC are the preprocessing systems of the video signal. The block diagram considered is shown in Fig. (1). It includes thermal sensors, analog circuits, and microprocessor. The output of the processed signal satisfies RS-170 video standard. The sensor elements for thermal imaging is $256 \times 256$ focal plane $\mathrm{CCD}[1-3]$, the gray level of each element is digitalized to be 256 states with 8bit A/D converter. Fig. (2) shows typical minimal and maximal gray level of a thermal picture. Analog circuit gives faster signal amplification in forward-loop. It needs $256 \times 256 \times 60 \times 10 \mathrm{~Hz}$ at least for $60 \mathrm{~Hz}$ picture

*Address correspondence to this author at the Department of Aeronautical Engineering, National Formosa University, 64, Wen Hua Road, Huwei, Yunlin, 63208, Taiwan; Tel: +886-5-631-5537; Fax: +886-5-631-2415; Email: tstsay@nfu.edu.tw updating frequency ; i.e., greater than $39.32 \mathrm{MHz}$. Microprocessor providing thermal cell addressing for sampling and storing CCD sensing data, maximal, minimal, and average values calculations of the whole picture and digital filters for AGC and APC loops.

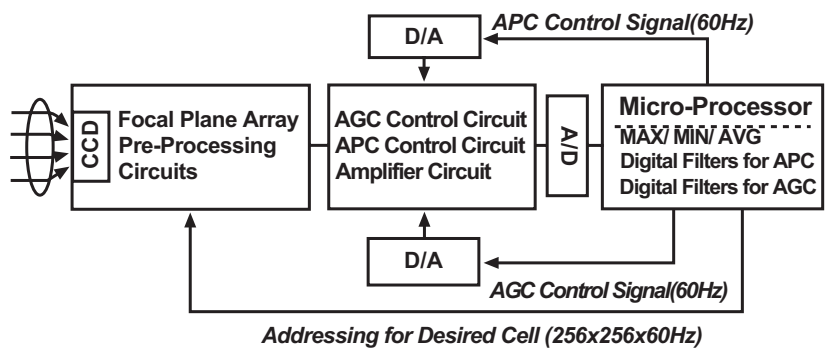

Fig. (1a). Block diagram of the data pre-processing.

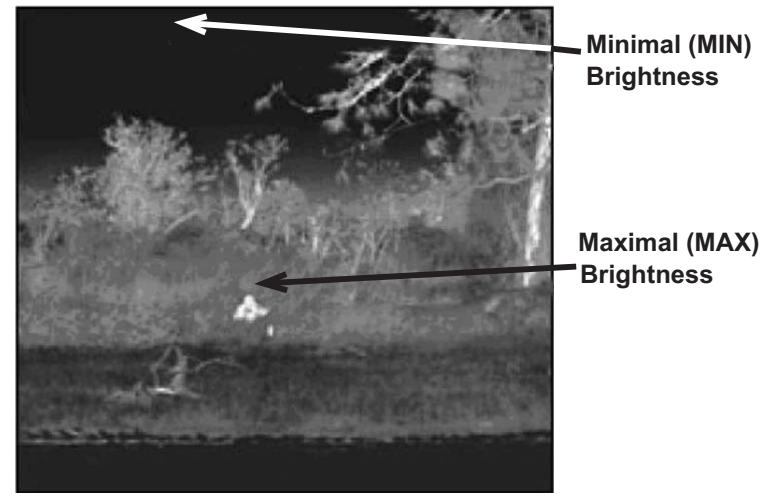

Fig. (1b). The minimal and maximal Brightness of a thermal picture.

Fig. (2) shows the analog circuit component of the overall system. The input is "Signal" form CCD and output is digitalized with AD converter ADC208, the "Gain" is the connection for AGC with $60 \mathrm{~Hz}$ updating rate. The "Offset" is the connection for APC with $60 \mathrm{~Hz}$ updating rate, the "Extern" is the selection for APC/AGC and manual adjustment 
and the "Clock" and "ADClock" are clocking for sampleddata and analog to digital conversation.

Combing the digital circuit, the overall system can be described by a nonlinear sampled-data feedback control system $[8,9]$. Fig. (3) shows the system. The gray blocks are models for analog circuit, and the others are models for digital circuits. Fig. (3) shows two control loop for controlling contrast and brightness. The desired values are adjusted by $V_{p}$ and $V_{G}$. The detail description of the Fig. (3) is given below:

VCA is the voltage control amplifier (AD539), which is controlled by $V_{x}$. The relationship between input and output is in the form of

$$
V_{o 1}=V_{x}\left(V_{y 2}-V_{y 1}\right)
$$

Where $V_{y 2}$ is the output voltage of CCD sensor element; $V_{y 1}$ is output of the APC loop; $V_{x}$ is the output of the AGC loop; and $V_{\text {agco }}$ is the offset value of $V_{x}$ for preventing exact blanking of input signals $V_{i 1}$. Note that the exact blanking will make the AGC loop operates at opened and closed bi-state condition. $K_{o}$ is the feed-forward loop gain; $V_{G}$ is the desired video contrast; and $V_{p}$ is the desired video brightness. The transducer [1-3] of the system is represented by a high pass transfer function, in which $K_{i}$ and $a$ are gain and dynamic of it; respectively. The $\Delta T$ is the relative temperature difference of the thermal source with respect to the background. The feedback signal of the AGC control loop is MAX-MIN. The feedback signal of the APC loop is AVG. $N_{1}$ and $N_{2}$ represent limitations of digital to analog converters; $N_{3}$ is the limitation for satisfying the $R S-170$ video standard. The desired digital controllers are $G_{1}(z), G_{2}(z)$ and $1 / 0.6\left(V_{x o}+V_{a g c o}\right)$. Analog parts of each loop are used to washout noise and effects of quantization error of digital control signals; especially for larger signals (i.e., smaller value of $V_{x}$ ). Note that bandwidths of AGC and APC loops must fast enough to suppress $\Delta T$ increasing for preventing the output $V_{o}$ be saturated.

It is difficult to model inherent nonlinearities MIN, MAX, and AVG in the overall AGC and APC system simultaneously and perform frequency responses to get suitable digital compensations for coping with approaching behaviors between seeker and target. One possible way for this problem is to decompose the overall AGC and APC control system into two individual control loops: one is the APC loop with the disturbance from the AGC loop; another is the AGC loop. The AGC loop is not affected by applying the APC loop for the use of the MAX-MIN as the feedback signal, which will not be affected by the average of video outputs. The average of video outputs can further be simplified as unity for analyzing the APC loop; and the contrast MAXMIN can also be simplified as unity for analyzing AGC loop. These decompositions and simplifications make the system can be analyzed and designed by conventional techniques for control systems. These simplifications will be verified by digital simulations and real flight testing.

In following sections, the overall system first decomposed into AGC and APC loops to find loop compensations; respectively, and then verified by digital simulations of the overall AGC/APC system with a special range-dependent testing signal, and finally verified by a thermal plate and real

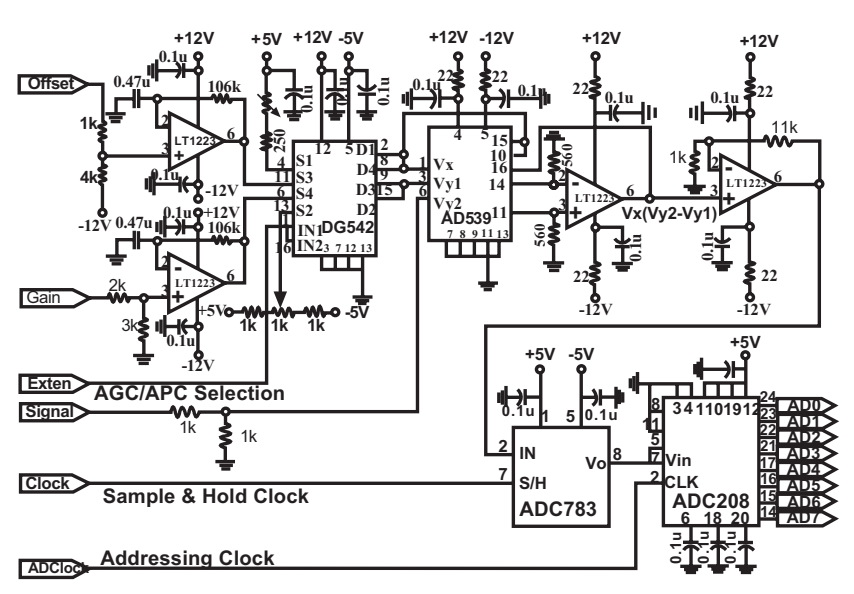

Fig. (2). Analog circuit for data pre-processing.

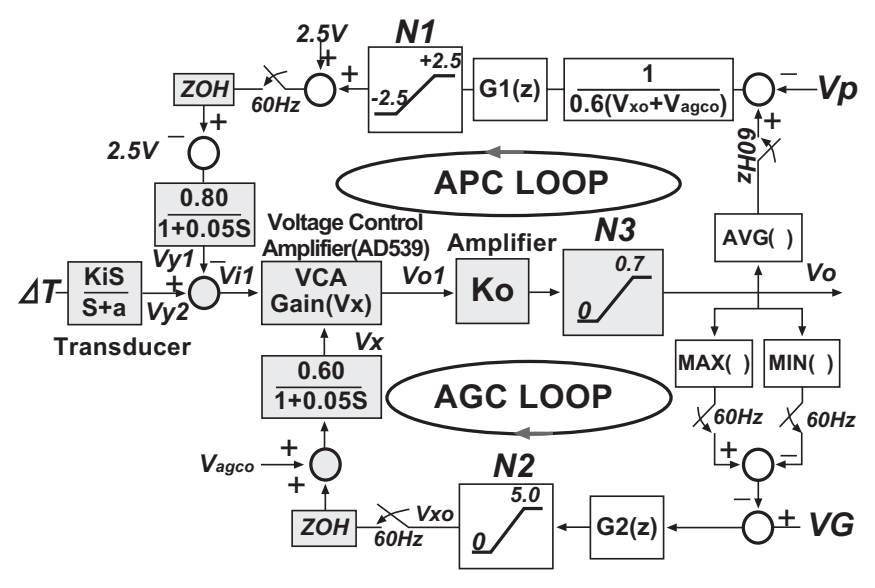

Fig. (3). The mathematical model of AGC/APC loops.

testing. From digital simulation and real testing results, it will be seen that the proposed method gives good approximations and provides an effective way for analyzing and designing the considered system.

\section{ANALYSES AND DESIGNS OF THE AGC LOOP}

Fig. (4) shows the simplified AGC loop, in which value of MAX-MIN can be further replaced by unity for finding steady-state solutions and compensation. These simplifications are suitable for slow variations of MAX and MIN, and the control of contrast(MAX-MIN) is equivalent to control video output $V_{o}$ for MIN can be set to be zero and MAX replaced by MAX-MIN; i.e., equivalent to level shifting. This assumption is correct for the location of MAX measured represents generally the location of the target and MIN represents the background. This simplified block diagram can be used to find the operating range of the AGC loop. The operating range is limited by characteristics of the voltage control amplifier (VCA), the feed-forward gain $K_{o}$, the desired contrast $V_{G}$, and the minimal control voltage $V_{\text {agco }}$ of VCA. Fig. (4) shows the active range of the AGC loop is the value of steady-state control voltage $V_{x}$ falls in $0.6 V_{a g c o}$ and $3 \mathrm{~V}$, which represents minimal and maximal gains of VCA are $0.6 V_{a g c o}$ and $3 \mathrm{~V}$; respectively. The corresponding levels of input signals are large and small; respectively. 


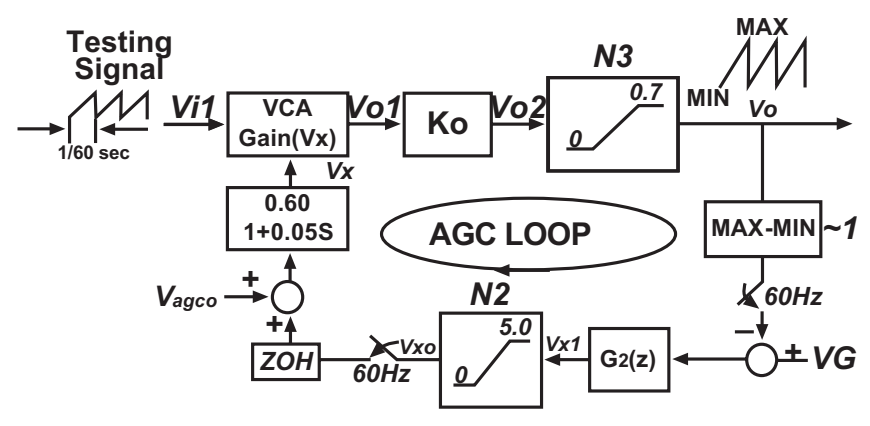

Fig. (4). The AGC loop and linearization for MAX-MIN.

The input/output relationship of each block in steadystate condition given in Fig. (4) can be written by following equations:

$$
\begin{aligned}
& V_{o 2}=K_{o} V_{i 1} V_{x} \\
& V_{o}=N_{3}\left(V_{o 2}\right) \\
& V_{X 1}=\left.G_{2}(z)\right|_{z=1}\left(V_{G}-V_{o}\right) \\
& V_{X o}=N_{2}\left(V_{X 1}\right) \\
& V_{x}=0.6\left(V_{X o}+V_{\text {agco }}\right)
\end{aligned}
$$

Note that the value $V_{i 1}$ is the maximal value of the input signal MAX in the sampling interval. From Equations (2) to (6), one has

$$
V_{x}=0.6\left\{N_{2}\left(\left.G_{2}(z)\right|_{z=1}\left[V_{g}-N_{3}\left(K_{o} V_{i 1} V_{x}\right)\right]+V_{a g c o}\right\}\right.
$$

The steady-state solution $V_{x s}$ (and $V_{o s}$ ) can be found by Equation(7) for specified values of input signal $V_{i 1}$ and command $V_{G}$. Since the values of $V_{x}$ is limited in [0.6 $\mathrm{V}_{\text {agco }}$, $3 \mathrm{~V}]$, the found solution $V_{x s}$ in $\left[0.6 V_{a g c o}, 3 \mathrm{~V}\right]$ represents the AGC loop is active for this specified value of $V_{i 1}$. If there is no solution found, the value of $V_{x s}$ will be at $0.6 V_{\text {agco }}$ for large value of $V_{i 1}$ or at $3 \mathrm{~V}$ for small value of $V_{i 1}$. Note that steady-state conditions exist only for stable system. Therefore, the $G_{2}(z)$ must be designed not only for performance but also for stability.

Fig. (5) shows solutions of solving Equation (7) for $V_{G}=0.5 \mathrm{~V}, K_{o}=12, G_{2}(1)=100, V_{a g c o}=0.05 \mathrm{~V}$, and some specified values of $V_{i 1}$. It represents the AGC loop is active for magnitudes of input signal $V_{i 1}$ between $\mathrm{A}$ and B. Since the saturating level of $N_{3}$ is $0.7 \mathrm{~V}$, magnitudes of input signal between $\mathrm{B}$ and $\mathrm{C}$ are still useful. Beyond $\mathrm{C}$, signals will be distorted by introducing the saturating nonlinearity $N_{3}$. Fig. (5) represents the operating range of input signal of the AGC loop; i.e., the maximal acceptable and the minimal detectable signal inputs. Base upon the operating range found, one could determine the gains of transducer $K_{i}$ (e.g., gains of thermal-electrical device [1-3]) for a given maximal possible relative temperature difference of the target. $25 \mathrm{mV} /{ }^{\circ} \mathrm{C}$ is selected. On the other hand, one can adjust values of $K_{o}$ and $V_{\text {agco }}$ to get the suitable operating range for a given gain of transducer. The value of $V_{a g c o}$ determines the maximal acceptable signal input of the AGC loop (Point B given in Fig. 5), and the feed-forward loop gain $K_{o}$ determines the minimal detectable signal input (Point A given in Fig. 5).

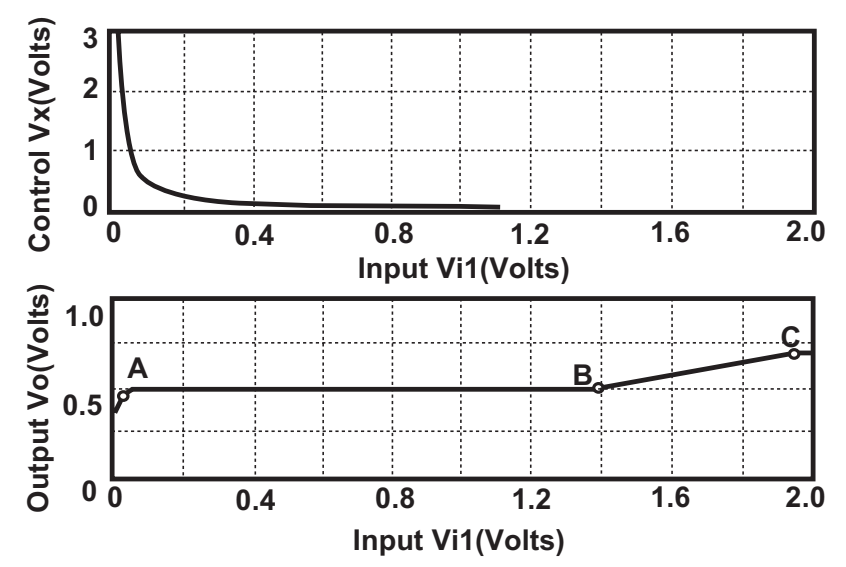

Fig. (5). Output and control gain versus input signal.

The Voltage Control Amplifier VCA can be replaced by $V_{i 1} V_{x s}$, in which $V_{x s}$ represents the steady-state solution of the AGC loop found by solving Equation (7) for a specified value of $V_{i 1}$. The transfer function of form $V_{G}$ to $V_{o}$ can be written as

$$
V o(z)=\frac{K_{o} G_{2}(z) Z\left\{\frac{0.6}{1+0.01 s}\right\} V_{i 1 s} V_{x s}}{1+K_{o} G_{2}(z) Z\left\{\frac{0.6}{1+0.01 s}\right\} V_{i 1 s} V_{x s}} V_{G}(z)
$$

where $\mathbf{Z}\{\bullet\}$ is operator of the z-transformation. Equation (8) gives if the AGC loop was in active region(between point $\mathrm{A}$ and point B shown in Fig. 5); frequency responses given by Equation(8) will keep the same even for different input values of $V_{i 1}$. Therefore, the output $V_{o}$ will track command $V_{G}$. Equation (8) can be used also to find digital controller $G_{2}(z)$ for wanted performance and robustness. The found digital controller $[10,11]$ for phase margins greater than $60^{\circ}$ and gain margin greater than $6 \mathrm{dBs}$ is in the form of

$$
G_{2}(\mathrm{z})=\left.100 \frac{1+0.02 S}{1+2.00 S}\right|_{S=\frac{2(Z-1)}{T_{s(}(Z+1)}}=\frac{z-0.1700}{z-0.9917}
$$

where $T_{S}$ is equal to $1 / 60$ seconds [4]. $T_{S}$ is called frame time also. Fig. (6) shows open-loop Bode diagrams with values of input signal $V_{i 1}$ varying from $0.1 \mathrm{~V}$ to $1.3 \mathrm{~V}$. It gives phase margin $67.5^{\circ}$ and gain margin 26dBs. The frequency responses show the compensated system is robust against the variation of input signal $V_{i 1}$ in the active range. The corresponding steady state values of $V_{x}$ and $V_{o}$ are given in Table 1.

The bandwidth of the AGC loop is about $3.0 \mathrm{~Hz}$. The suitability of $3.0 \mathrm{~Hz}$ will be verified by time responses with input signal increasing. The phase margin $\mathrm{PM}=67.5^{\circ}$ is equivalent to pure time delay $\left(T_{d}=91.85 \mathrm{~ms}\right)$ in loop, and gain crossover frequency $\left(f_{n}=2.04 \mathrm{~Hz}\right)$ are all given in Fig. (6). The phase margin will be verified by digital simulations with equivalent pure time delay $\left(T_{d}\right)$; i.e., $V_{x o}$ is replaced by $V_{x o} e^{-T_{d} S}$.

Fig. (7) shows time responses of the AGC loop with digital controller $G_{2}(z)$ for $V_{G}=0.5 \mathrm{~V}$. The input testing signal is 
Table 1. Calculated and Simulated Results for Different Inputs

\begin{tabular}{|c|c|c|c|c|}
\hline Inputs & \multicolumn{2}{|c|}{ Calculated Results } & \multicolumn{2}{c|}{ Simulated Results } \\
\hline \hline$V_{i 1}$ & $V_{x s}$ & $V_{o s}$ & $V_{x}$ & $V_{o}$ \\
\hline 0.01 & 3.00 & 0.360 & 3.00 & 0.398 \\
\hline 0.05 & 0.811 & 0.487 & 0.740 & 0.489 \\
\hline 0.10 & 0.411 & 0.493 & 0.376 & 0.494 \\
\hline 0.30 & 0.138 & 0.498 & 0.126 & 0.500 \\
\hline 0.50 & 0.083 & 0.500 & 0.075 & 0.500 \\
\hline 0.75 & 0.056 & 0.500 & 0.054 & 0.500 \\
\hline 1.00 & 0.042 & 0.500 & 0.038 & 0.500 \\
\hline 1.25 & 0.033 & 0.500 & 0.030 & 0.503 \\
\hline 1.50 & 0.030 & 0.540 & 0.030 & 0.569 \\
\hline 2.00 & 0.030 & 0.700 & 0.030 & 0.700 \\
\hline
\end{tabular}

a saw-tooth wave shown in Fig. (4) and maximums in sampling intervals are increasing with a time function in the form of

$$
F(t)=\left(\frac{\gamma}{\beta-t}\right)^{2}
$$

where $t$ is the simulation time, $\beta=5.238$, and $\gamma=0.3704$. The value 5.238 represents $1.3 \mathrm{Km}$ for missile speed equal to $250 \mathrm{~m} / \mathrm{s}$. The control voltage $V_{x}$ of VCA is decreasing for the input signal $V_{i 1}$ is increasing with the time function described by Equation (10), and the output $V_{o}$ keeps almost constant $(\sim 0.5 \mathrm{~V})$ in the active range. It can be seen that $V_{x}$ keeps its maximal value $(3 \mathrm{~V})$ in the inactive range (before 2 seconds), and $0.6 V_{\text {agco }}$ after 4.8 seconds. Fig. (7) shows the closed-loop system compensated by $G_{2}(z)$ is suitable for given target approaching behaviors. A stop homing range $\sim 250 \mathrm{~m}$ is usually selected for RF seeker used only. It prevents the saturation of the RF receiver. The less stop homing range, the less miss distance will be. Fig. (7) shows that the shorter stop homing range can be selected for less miss distance.
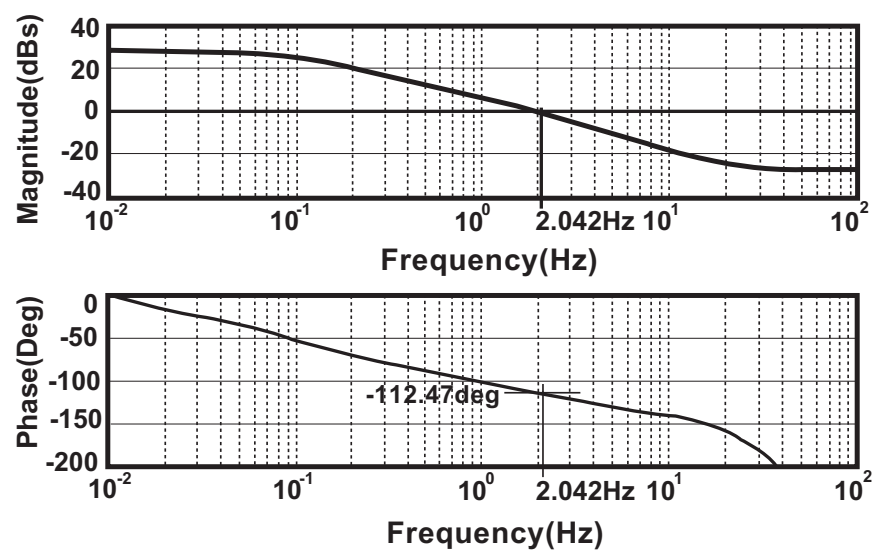

Fig. (6). Open-loop Bode diagram of AGC $\operatorname{loop}\left(V_{i 1}=0.1 \sim 1.3\right)$.
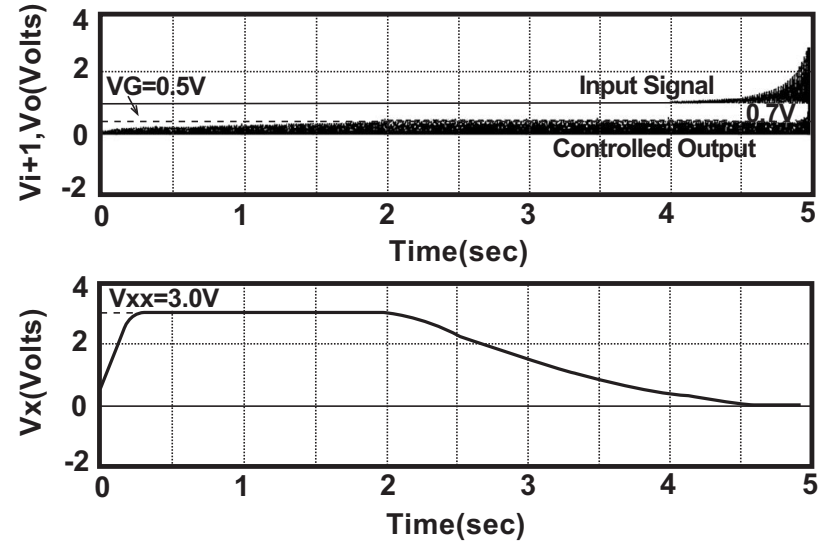

Fig. (7). Time responses for subsonic approaching.

Note that the feedback signal of the AGC loop is MAXMIN, thus saw-tooth wave can be used to simulate maximums and minimums of real input signals in sampling intervals for there is no frequency element in the feed-forward signal loop(i.e., from $V_{i 1}$ to $V_{o}$ ). Fig. (7) shows active, inactive, and saturating characteristics of the AGC loop (c.f. Fig. 5). Several constant-input $V_{i 1}$, digital simulations are made also to verify calculated results from the simplified AGC loop. The simulated and calculated results are given in Table 1. It can be seen that calculated results give good agreements with simulated results. Note the magnitude of $V_{i l}$, stated in steady-state analyses is equivalent to maximal amplitude of the saw-tooth wave in sampling interval in digital simulations.

The stability [12,13] of the AGC loop compensated by Equation (9) can be verified by introducing the maximal acceptable pure time delay $\left(T_{d}\right)$ into loop in digital simulation. Note that the maximal acceptable pure time delay represents the system will become unstable by introducing pure time delay greater than $T_{d}$. Fig. (8) shows the stability testing for the maximum of input signal $V_{i 1}$ equal to $0.05 \mathrm{~V}$ with $6 / 60$ seconds pure time delay into the position between the digital control signal $V_{G}-\mathrm{MAX}+\mathrm{MIN}$ and $G_{2}(z)$. The oscillating frequency of the control signal $V_{x}$ is $1.85 \mathrm{~Hz}$, which is close to the found gain crossover frequency given in Fig. (6) with the value of MAX-MIN is set to be unity. This implies that the simplification is satisfactory. The relationship between the maximal acceptable pure time delay $\left(T_{d}\right)$ in second, phase margin (PM) in degree, and oscillating frequency $\left(f_{n}\right)$ in Hertz is in the form of

$$
\mathrm{PM}=360 f_{n} T_{d} \mathrm{deg} .
$$

Based upon Equation (11), the found phase margin is $65.5^{\circ}$ in the above constant- $V_{i 1}$ stability testing. It is closed to that of the analyzed result shown in Fig. (6).

Fig. (9) shows time responses with $T_{d}=6 / 60$ seconds pure time delay in loop, and magnitudes of input signal $V_{i 1}$ are increasing with the time function described by Equation(10). It can be seen that the phase margin of the AGC loop is greater than $60^{\circ}$ for the maximal acceptable loop pure time delay $T_{d}=6 / 60$ seconds and the oscillating frequency $f_{n}=$ 2.0Hz. The results shown by Figs. (8) and (9) represent dy- 
namics of the compensated system are close to results of analyses with the simplification of the AGC loop. Note that it is need not find the gain margin of the AGC loop for its object is to adjust loop gain.
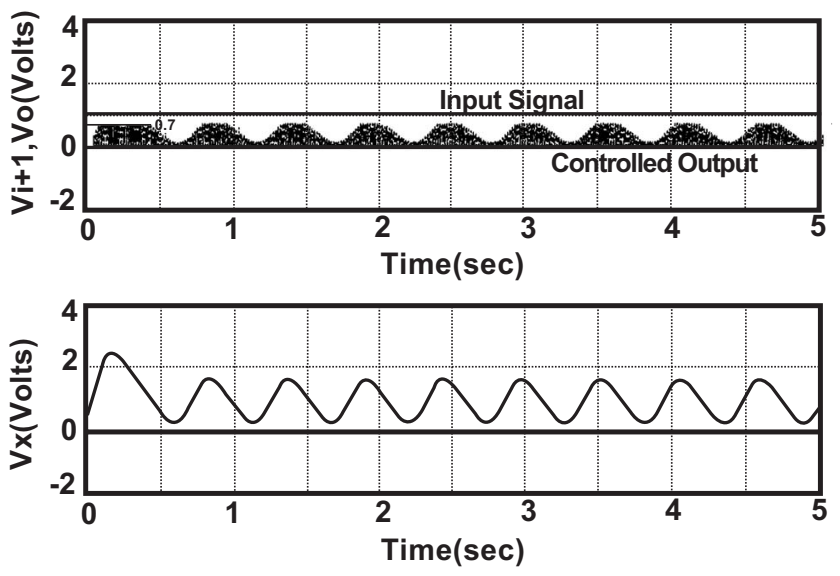

Fig. (8). Simulating verification for AGC loop $(1.85 \mathrm{~Hz})$ for $V_{i 1}=0.05 \mathrm{~V}$ and $T_{d}=100 \mathrm{~ms}$.
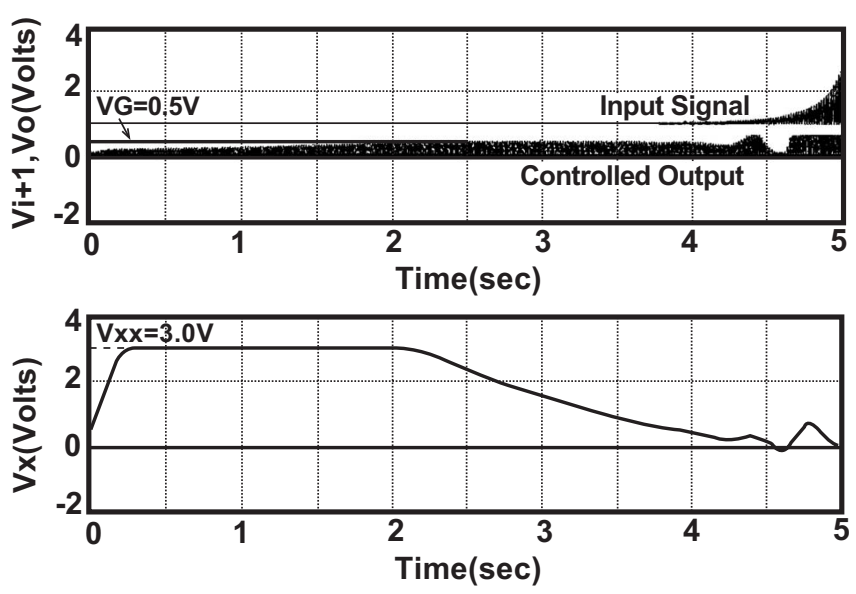

Fig. (9). Simulating verification for AGC loop for varying input and $T_{d}=100 \mathrm{~ms}$.

\section{ANALYSES AND DESIGNS OF THE APC LOOP}

Fig. (10) shows the APC loop, in which $G_{1}(z)$ and $1 / V_{x}$ are digital controllers; $V_{y 2}$ represents the disturbance of the APC loop from input signals; AVG is set to be unity also; voltage control amplifier(VCA) is replaced by its control voltage $V_{x}$. The use of $1 / V_{x}$ is to keep constant loop gain and linearize the APC loop. The values of $V_{x}$ is determined by the AGC loop and input signal $V_{i 1}$. The found digital controller of the APC loop is given below:

$$
G_{I}(z)=\left.\frac{T s(z+1.000)}{2(z-0.997)} \cdot 1.25 \frac{1+0.05 S}{1+0.005 S}\right|_{S=\frac{2(Z-1)}{T_{s(Z+1)}}}
$$

The bandwidth of the APC loop is about $2.4 \mathrm{~Hz}$. The gain/ phase margins and phase/ gain crossover frequencies are $\mathrm{PM}=81.1^{\circ}$ with gain crossover frequency $f_{n}=1.9 \mathrm{~Hz}$ and $\mathrm{GM}=10.1$ with phase crossover frequency $f_{n}=17.45 \mathrm{~Hz}$. The verifications for gain/phase margins will be discussed with overall system for $1 / V_{x}$ is used in APC loop shown in Fig. (10).

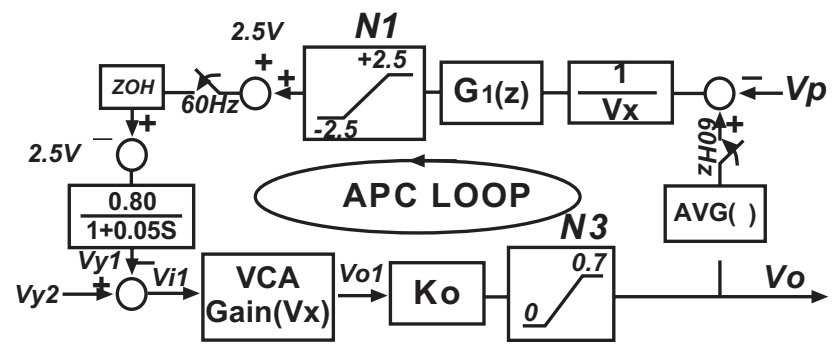

Fig. (10). The APC loop with disturbance $V_{y 2}$.

\section{DIGITAL SIMULATIONS AND VERIFICATIONS OF THE OVERALL SYSTEM}

Fig. (11) shows time responses of the overall system for $V_{P}=0.35 \mathrm{~V}$ and $V_{G}=0.5 \mathrm{~V}$ with the range-dependent input signal $V_{y 2}$ described by Equation(10). It shows operating behaviors of AGC and APC loops. For instance, the output signal $V_{O}$ before 2 seconds keeps wanted average at $V_{p}=0.35 \mathrm{~V}$ with maximal control voltage $V_{x}=3 \mathrm{~V}$. The contrast cannot follow $V_{G}$ for magnitudes of input signals $V_{i 1}$ are too small (c.f., below Point A given in Fig. 5). After 2 seconds, the AGC loop is active, the contrast keep desired value $V_{G}=0.5 V$ until $V_{x}$ decreased to be $0.6 V_{a g c o}$. Comparing results given in Fig. (7) without the APC, it can be seen that the overall system provides constant brightness with the desired contrast similar to results given in Fig. (7).
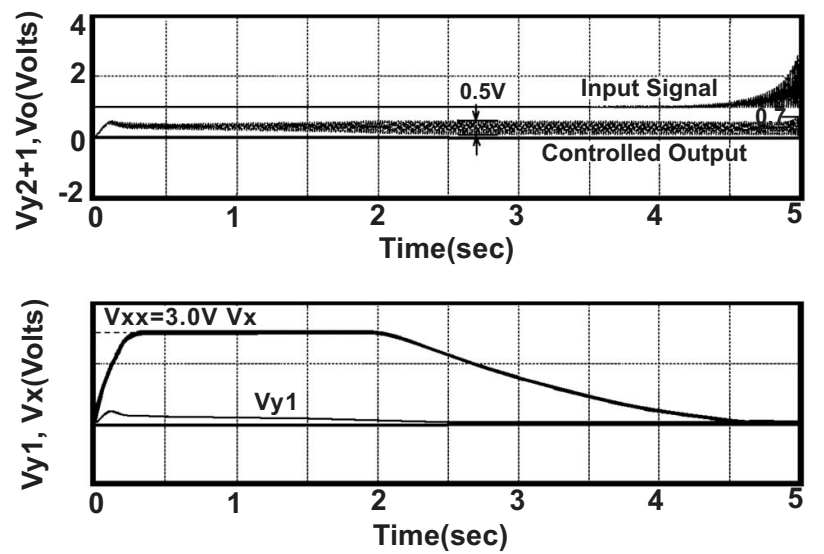

Fig. (11). Time responses for overall AGC/APC loop.

Figs. (12) and (13) show results of stability testing of the APC loop for finding gain and phase margins in digital simulations of the overall system. Fig. (12) shows results of the APC control loop with pure time delay $T_{d}=7 / 60$ seconds; Fig. (13) shows results of the APC control loop gain multiplied by 10.1 . The equivalent phase margin (PM) can be found by Equation (11). The phase margin is greater than $80 \mathrm{deg}$ for the maximal acceptable loop pure time delay $T_{d}=7 / 60$ seconds and the oscillating frequency $f_{n}$ of $V_{y 2}$ is about $2 \mathrm{~Hz}$. It can be seen that the simplified APC loop shown in Fig. (1) provides a good approximation also for the real system shown by Fig. (3). Therefore, simplifications of two individual loops are satisfactory for analyses and designs of the complicated nonlinear sampled-data control system. 

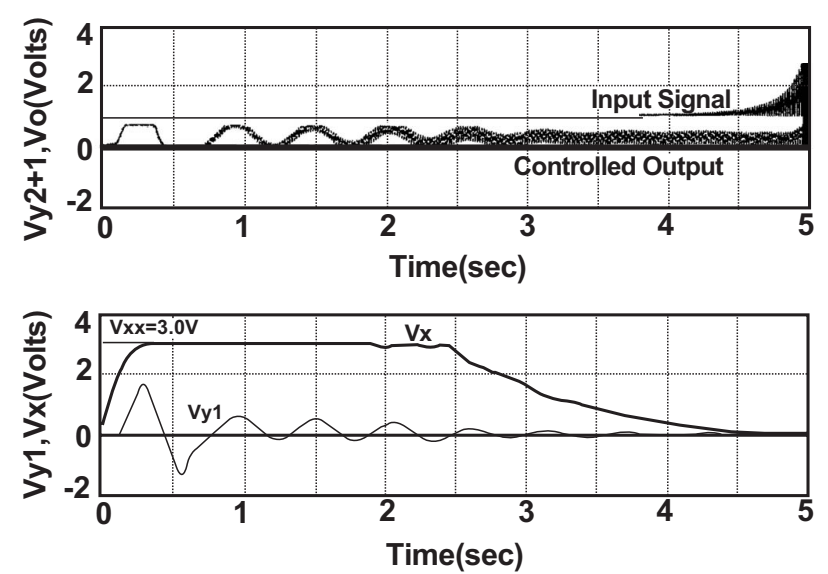

Fig. (12). Time responses of the overall system with $T_{d}=7 / 60$ " in APC loop.

Fig. (14) shows the thermal testing plate for target approaching phase. It is made by light emitter diodes (LEDs). The distance between image seeker and thermal plate is 5 meters and dimension of thermal plate is $120 \mathrm{~cm} \times 88 \mathrm{~cm}$ for field of view(FOV) of the image seeker is $14^{\circ} \times 10^{\circ}$. The dimension of ship-shape is increased with time function described by Equation (10). The testing results gives the function design of AGC/APC circuit is valid for anti-ship applications. Fig. (15) shows the typical thermal image of target on flight testing. The distance between imager and ship is $410 \mathrm{~m}$.
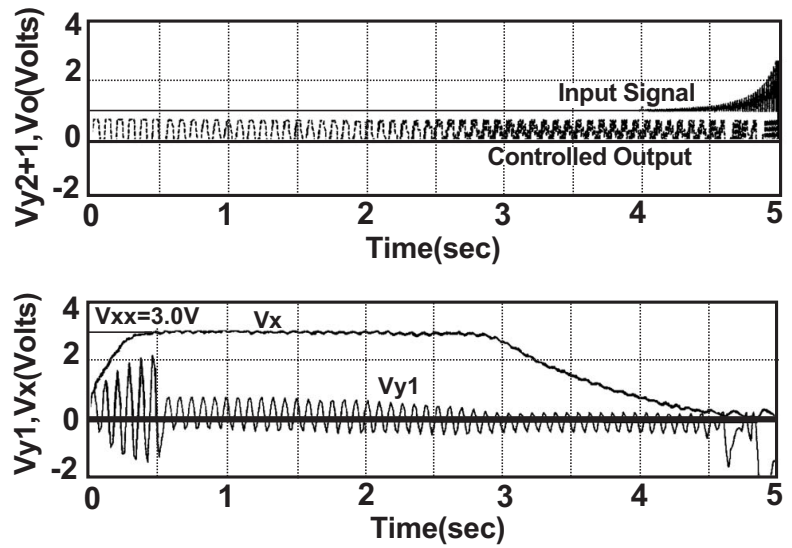

Fig. (13). Time responses of the overall system with Gain=10.1 in the APC loop.

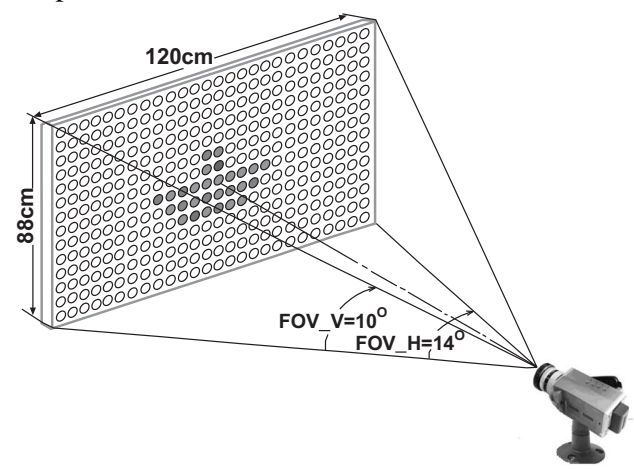

Fig. (14). Thermal plate testing concepts for target approaching phase.

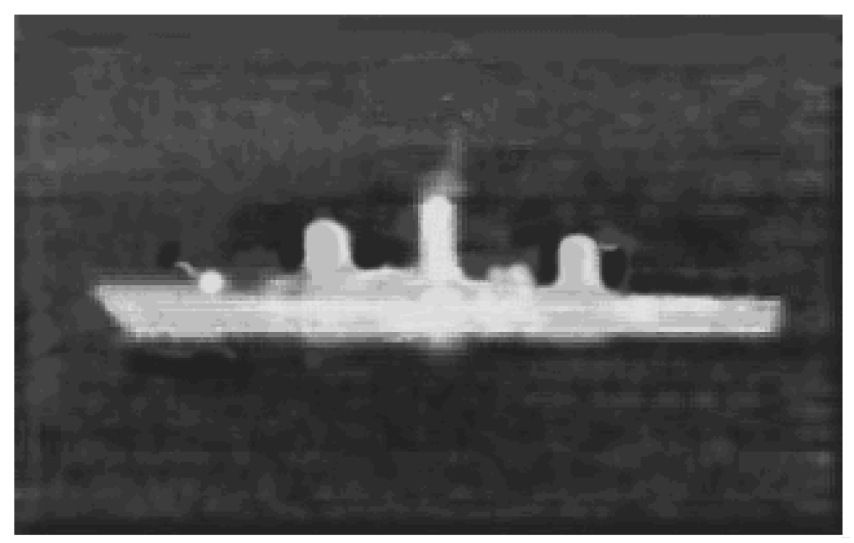

Fig. (15). Thermal image of target in the terminal target approaching phase.

\section{CONCLUSIONS}

In this literature, a data pre-processing circuit designs and analyses for an infrared image seeker of a subsonic cruise missile have been proposed. The overall system is a complicated nonlinear sampled-data control system. It was first decomposed into two linearized sampled-data control systems to get desired loop compensations, and verified with a special range-dependent testing signal for the overall system secondly. The controlled system was further verified by a thermal plate and real flight testing. Simulation and testing results gave the proposed method can provide effective way to analyze and design the considered system.

\section{REFERENCES}

[1] D.F. Barbe, "Imaging Devices Using the Charge-Coupled Concepts", Proc. IEEE, 63(1): 38-67, 1975.

[2] C.H. Sequin and M.F. Tompsett, Charge Transfer Devices, Academic Press Inc. 1975.

[3] F.D. Shephard, R.W. Taylor, B.R. Skolnik, et al., "Schottky IRCCD Thermal Imaging, Advances in Electronics and Electron Physics", 1979, 52, 7th Symposium on Photo- Electronic Image Devices.

[4] W.N. Lie, "Automatic Camera Gain and Pedestal Control for Adaptive Scene Contrast Enhancement', J. Electronic Imaging, 1: 244-51, 1992.

[5] K.R. Fowler, "Automatic Gain Control for Image-Intensified Camera", IEEE Trans. Instrum. Meas., 53(4): 1057-64, 2004.

[6] J Castracane and M. Gutin, "DMD-based Bloom Control for Intensified Imaging Systems, Proc. SPIE, 3633: 234-42, 1999.

[7] B.R. Dobbie, "Development of a Night Vision Device Incorporating an Integral Video Display, Increase Field of View, and Advanced Intensifier Gain Features", Proc. SPIE, 3173: 500-21, 1997.

[8] R. W. Newecomb, Nonlinear system analyses, Prentice-Hall International Inc., New Jersey, 1978.

[9] J.J.E. Slotine and Li W., Applied nonlinear control, Prentice-Hall International Inc., New Jersey, 1991.

[10] G.F. Franklin, J.D. Powell, A. Emami-Naeini. Feedback Control of Dynamics, Addison-Wesley Publishing Company, 1986.

[11] K. Ogata, Discrete-Time Control System, Prentice-Hall Inc. Englewood Cliffs, NJ, 1994.

[12] D.N. Green, "Global Stability Analysis of Automatic Gain Control Circuits", IEEE Trans. Circuits Syst., 30(2): 78-83, 1983.

[13] D.M. Badger, "Stability of AGC Circuits Containing Peak Detectors", IEEE Trans. Consumer Electronics, 38(3): 377-83, 1992. 